DF/IST-3.99

OCTOBER 1999

gr-qc/yymmnn

\title{
Proposed astrophysical test of Lorentz invariance
}

\author{
O. Bertolamif and C.S. Carvalho \\ Departamento de Física \\ Instituto Superior Técnico, Av. Rovisco Pais \\ 1049 - 001 Lisboa, Portugal
}

\begin{abstract}
Working in the context of a Lorentz-violating extension of the standard model we show that estimates of Lorentz symmetry violation extracted from ultra-high energy cosmic rays beyond the Greisen-Kuzmin-Zatsepin (GZK) cutoff allow for setting bounds on parameters of that extension. Furthermore, we argue that a correlated measurement of the difference in the arrival time of gamma-ray photons and neutrinos emitted from active galactic nuclei or gamma-ray bursts may provide a signature of possible violation of Lorentz symmetry. We have found that this time delay is energy independent, however it has a dependence on the chirality of the particles involved. We also briefly discuss the known settings where the mechanism for spontaneous violation of Lorentz symmetry in the context of string/M-theory may take place.
\end{abstract}

PACS: 98.70.Sa, 04.80.CC, 11.30.Cp, 98.70.Rz

\footnotetext{
${ }^{1}$ E-mail address: orfeu@cosmos.ist.utl.pt
} 


\section{Introduction}

Lorentz invariance is one of the most fundamental symmetries of physics and underlies all known physical descriptions of nature. However, more recently, there has been evidence, in the context of string/M-theory, that this symmetry could, at least in principle, be spontaneously broken. This raises immediately the issue of investigating this possibility from the experimental point of view. Observational information on the violation of Lorentz symmetry may, of course, provide essential insights into the nature of the fundamental theory of unification and hopefully allow establishing relevant bounds on its parameters.

In this work, we shall argue that astrophysics may play an essential role in this respect. This comes about as it will soon be possible to make correlated astrophysical observations involving high-energy radiation and neutrinos. Indeed, it is remarkable that there exists convincing evidence that the observed jets of active galactic nuclei (AGN), powered by supermassive black holes at their core, are quite efficient cosmic proton accelerators. The photoproduction of neutral pions by accelerated protons are assumed to be the source of the highest-energy photons through which most of the luminosity of the galaxy is emitted. The decay of charged pions with the ensuing production of neutrinos is another distinct signature of the proton induced cascades [1]. Moreover, there is a consensus that estimates of the neutrino flux are fairly model independent and that reliable upper bounds can be established [2]. Since gamma-ray bursts (GRB) have also been suggested as a possible source of high-energy neutrinos [3] the mentioned upper bounds are also valid for those sources. To further deepen the knowledge of these sources and also because the cosmicray energy spectrum extends to energies higher than $10^{20} \mathrm{eV}$, large area $\left(\sim \mathrm{km}^{2}\right)$ high-energy neutrino telescopes are under construction (see e.g. [4]). These telescopes will allow for obtaining information that is intrinsically correlated with the gamma radiation emitted by AGN and GRB. On the other hand, it has already been pointed out that astrophysical observations of faraway sources of gamma radiation could provide important hints on the nature of gravity-induced wave dispersion in vacuum [5, 6, 0, 8] and hence on physics beyond the standard model (SM). In here, we will show that delay measurements in the arrival time of correlated sources of gamma radiation and high-energy neutrinos can, when considered in the context of a Lorentz-violating extension 
of the SM [9], help in setting a relevant limit on the violation of that fundamental symmetry. We shall further relate our results with the recently discussed limit on the violation of Lorentz symmetry from the observations of high-energy cosmic rays beyond the Greisen-Kuzmin-Zatsepin (GKZ) cutoff [10].

However radical, the idea of dropping the Lorentz symmetry has been repeatedly considered in the literature. Indeed, a background or constant cosmological vector field has been suggested as a way to introduce our velocity with respect to a preferred frame of reference into the physical description [11]. It has also been suggested, based on the behaviour of the renormalization group $\beta$ function of non-Abelian gauge theories, that Lorentz invariance could be just a low-energy symmetry [12]. Furthermore, higher-dimensional theories of gravity that are not locally Lorentz invariant have been considered in order to obtain light fermions in chiral representations [13].

The breaking of Lorentz symmetry due to nontrivial solutions of string field theory was first discussed in Refs. [14]. These nontrivial solutions arise in the context of the string field theory of open strings and may have striking implications at low-energy. For instance, assuming that the contribution of Lorentz-violating interactions to the vacuum energy is about half of the critical density was shown to allow concluding on the existence of quite feeble tensor mediated interactions in the range of about $10^{-4} \mathrm{~m}$ [15]. Furthermore, Lorentz violation may be a factor in the breaking of conformal symmetry and this together with inflation may lie at the origin of the primordial magnetic fields which are required to explain the observed galactic magnetic field [16]. Of course, putative violations of the Lorentz invariance may contribute to in the breaking of CPT symmetry [17]. Interestingly, this possibility can be verified experimentally in neutral-meson [18] experiments? Penning-trap measurements [20] and hydrogen-antihydrogen spectroscopy [21]. Moreover, the breaking of CPT symmetry also allows for an explanation of the baryon asymmetry of the Universe. Tensor-fermion-fermion interactions expected in the low-energy limit of string field theories give rise in the early Universe, after the breaking of the Lorentz and CPT symmetries, to a chemical potential that creates in equilibrium a baryon-antibaryon asymmetry in the presence of baryon number violating interactions [22].

\footnotetext{
${ }^{2}$ These CPT violating effects are unrelated to those that are due to possible nonlinearities in quantum mechanics, presumably arising from quantum gravity, which were already investigated by the CPLEAR Collaboration [19].
} 
Limits on the violation of Lorentz symmetry have been directly sought through laser interferometric versions of the Michelson-Morley experiment, where comparison is made between the velocity of light, $c$, and the maximum attainable velocity of massive particles, $c_{i}$, up to $\delta \equiv\left|c^{2} / c_{i}^{2}-1\right|<10^{-9}$ [23]. More accurate tests can be performed via the so-called Hughes-Drever experiment [24, 25]. In the latter type of measurement, one searches for the time dependence of the quadrupole splitting of nuclear Zeeman levels along Earth's orbit and that allows for the achievement of impressive limits, for instance, $\delta<3 \times 10^{-22}$ [26]. Actually, a more recent assessment of these experiments reveals that more stringent bounds, up to eight orders of magnitude, can be reached [27]. From the astrophysical side, limits on the violation of momentum conservation and the existence of a preferred reference frame can also be established from bounds on the parametrized post-Newtonian parameter, $\alpha_{3}$. This parameter vanishes identically in general relativity and can be accurately determined from the pulse period of pulsars and millisecond pulsars [28, 29]. The most recent limit, $\left|\alpha_{3}\right|<2.2 \times 10^{-20}$ [30], indicates that Lorentz symmetry holds up to this level.

In what follows we shall compute the corrections to the dispersion relation arising from a Lorentz-violating extension of the SM and confront it with the evidence on the violation of Lorentz invariance as arising from cosmic ray physics. Moreover, we shall show that these corrections induce a time delay in the arrival of signals carried by different particles from faraway sources. We also find that this time delay is energy independent, but it has a dependence on the chirality of the arriving particles.

\section{Lorentz-Violating Extension of the Standard Model and Dispersion Relation}

It is widely believed that the SM, although quite successful from the phenomenological viewpoint, is a low-energy approximation of a more fundamental theory where unification with gravity is achieved and the hierarchy problem solved. It is quite conceivable that, in this most likely higherdimensional underlying theory, fundamental symmetries, such as CPT and Lorentz invariance, may undergo spontaneous symmetry breaking. The fact that within string/M-theory, currently 
the most promising proposal for a fundamental theory, a mechanism where spontaneous breaking of Lorentz symmetry is known [14, 17, 31], indicates that the violation of those symmetries might actually occur and that its implications may be expected.

A priori, there is no reason for this breaking not to extend into the four-dimensional spacetime. If this is so, CPT and Lorentz symmetry violations will be likely to occur within the SM and its effects might be detected. In order to account for the CPT and Lorentz-violating effects an extension to the minimal $\mathrm{SU}(3) \otimes \mathrm{SU}(2) \otimes \mathrm{U}(1) \mathrm{SM}$ has been developed [9] based on the idea that CPT and Lorentz-violating terms might arise from the interaction of tensor fields to Dirac fields when Lorentz tensors acquire nonvanishing vacuum expectation values. Interactions of this form are expected to arise from the string field trilinear self-interaction, as in the open string field theory [14, 17]. These interactions may also emerge from the scenario where our world is wrapped in a brane and this is allowed to tilt [31]. Aiming to preserve power-counting renormalizability of the SM, only terms involving operators of mass dimension four or less are considered in this extention. In this work, only the fermionic sector of the extension discussed in [9] will be considered p]. This sector includes both leptons and quarks, since $\mathrm{SU}(3)$ symmetry ensures violating extensions to be colour-independent. The fermionic sector contains CPT-odd and CPT-even contributions to the extended Lagrangian, which are given by [9]

$$
\begin{gathered}
\mathcal{L}_{\text {Fermion }}^{\text {CPT-odd }}=-a_{\mu} \bar{\psi} \gamma^{\mu} \psi-b_{\mu} \bar{\psi} \gamma_{5} \gamma^{\mu} \psi, \\
\mathcal{L}_{\text {Fermion }}^{\text {CPT-even }}=\frac{1}{2} i c_{\mu \nu} \bar{\psi} \gamma^{\mu} \stackrel{\leftrightarrow}{\partial^{\mu}} \psi+\frac{1}{2} i d_{\mu \nu} \bar{\psi} \gamma_{5} \gamma^{\mu} \stackrel{\leftrightarrow}{\partial} \psi-H_{\mu \nu} \bar{\psi} \sigma^{\mu \nu} \psi,
\end{gathered}
$$

where the coupling coefficients $a_{\mu}$ and $b_{\mu}$ have dimensions of mass, $c_{\mu \nu}$ and $d_{\mu \nu}$ are dimensionless and can have both symmetric and antisymmetric components, and $H_{\mu \nu}$ has dimension of mass and is antisymmetric. All the Lorentz-violating coefficients are Hermitian. These parameters are flavour-dependent and some of them may induce flavour changing neutral currents when

\footnotetext{
${ }^{3}$ We shall suppose that the propagation features of photons are unaltered and hence that a Lorentz-violating extension of the SM gauge sector is unnecessary. Even though this possibility has been discussed in [9], the phenomenological restrictions are quite severe, at least in what concerns the term that gives origin to a cosmological birefringence [32].
} 
nondiagonal in flavour.

The Langrangian density of the fermionic sector including Lorentz-violating terms reads:

$\mathcal{L}=\frac{1}{2} i \bar{\psi} \gamma_{\mu} \stackrel{\leftrightarrow}{\partial^{\mu}} \psi-a_{\mu} \bar{\psi} \gamma^{\mu} \psi-b_{\mu} \bar{\psi} \gamma_{5} \gamma^{\mu} \psi+\frac{1}{2} i c_{\mu \nu} \bar{\psi} \gamma^{\mu} \stackrel{\leftrightarrow}{\partial^{\nu}} \psi+\frac{1}{2} i d_{\mu \nu} \bar{\psi} \gamma_{5} \gamma^{\mu} \stackrel{\leftrightarrow}{\partial^{\nu}} \psi-H_{\mu \nu} \bar{\psi} \sigma^{\mu \nu} \psi-m \bar{\psi} \psi$

where only kinetic terms are kept, as we are interested in deducing the free particle energymomentum relation.

From the above Lagrangian density, we can get the Dirac-type equation

$$
\left[i \gamma^{\mu}\left(\partial_{\mu}+\left(c_{\mu}^{\alpha}-d_{\mu}^{\alpha} \gamma_{5}\right) \partial_{\alpha}\right)-a_{\mu} \gamma^{\mu}-b_{\mu} \gamma_{5} \gamma^{\mu}-H_{\mu \nu} \sigma^{\mu \nu}-m\right] \psi=0
$$

In order to obtain the corresponding Klein-Gordon equation, we multiply Eq. (2) from the left by itself with an opposite mass sign yielding

$$
\begin{aligned}
& {\left[i\left(\partial_{\mu}+c_{\mu}^{\alpha} \partial_{\alpha}\right)-a_{\mu}\right]^{2}+\left(d_{\mu}^{\alpha} \partial_{\alpha}\right)^{2}-b^{2}-m^{2}-i \sigma^{\mu \rho}\left[i \partial_{\mu} c_{\rho}^{\beta} \partial_{\beta}+i c_{\mu}{ }^{\alpha} \partial_{\alpha} i \partial_{\rho}+i c_{\mu}{ }^{\alpha} \partial_{\alpha} i c_{\rho}{ }^{\beta} \partial_{\beta}\right.} \\
- & \left.i\left(\partial_{\mu}+c_{\mu}^{\alpha} \partial_{\alpha}\right) i d_{\rho}^{\beta} \gamma_{5} \partial_{\beta}+i d_{\mu}^{\alpha} \gamma_{5} \partial_{\alpha} i\left(\partial_{\rho}+c_{\rho}^{\beta} \partial_{\beta}\right)-2 b_{\mu} \gamma_{5}\left[i\left(\partial_{\rho}+c_{\rho}^{\beta} \partial_{\beta}\right)-a_{\rho}\right]\right] \\
- & 2 i\left(i a_{\mu} \sigma^{\mu \rho}-b_{\mu} \gamma_{5} g^{\mu \rho}\right) d_{\rho}^{\beta} \gamma_{5} \partial_{\beta}+\sigma^{\mu \nu} \sigma^{\rho \sigma} H_{\mu \nu} H_{\rho \sigma}-H_{\rho \sigma}\left(\gamma^{\mu} \sigma^{\rho \sigma}+\sigma^{\rho \sigma} \gamma^{\mu}\right)\left[i \left(\partial_{\mu}\right.\right. \\
+ & \left.\left.\left.\left(c_{\mu}^{\alpha}-d_{\mu}^{\alpha} \gamma_{5}\right) \partial_{\alpha}\right)-a_{\mu}+b_{\mu} \gamma_{5}\right]\right] \psi=0 .
\end{aligned}
$$

To eliminate the off-diagonal terms, the squaring procedure has to be repeated. However, as already discussed, since Lorentz symmetry breaking effects are quite constrained experimentally (see also [17, 15, 9] for theoretical discussions), violating terms higher than second order will be ignored. After some algebra, we find that off-diagonal terms cannot be fully eliminated, but that these terms are higher order in the Lorentz-violating parameters. To further simplify our computation we shall drop $H_{\mu \nu}$. This simplification is justifiable as in our phenomenological study we shall only consider the effect of the timelike components of the Lorentz-violating parameters. Hence, we obtain, for the Klein-Gordon type equation, up to second order in the new parameters:

$$
\begin{array}{r}
{\left[\left[(i \partial)^{2}+2 i \partial_{\mu} i c^{\mu \alpha} \partial_{\alpha}-2 i \partial_{\mu} a^{\mu}-m^{2}\right]^{2}+4 i \partial_{\mu} i d_{\rho}^{\beta} \partial_{\beta} i \partial_{\eta} i d_{\phi}^{\delta} \partial_{\delta}\left(g^{\mu \eta} g^{\rho \phi}-g^{\mu \rho} g^{\eta \phi}\right)\right.} \\
\left.-8 i \partial_{\mu} i d_{\rho}^{\beta} \partial_{\beta} b_{\eta} i \partial_{\phi}\left(g^{\mu \rho} g^{\eta \phi}-g^{\mu \phi} g^{\rho \eta}\right)+4 b_{\mu} b_{\eta} i \partial_{\rho} i \partial_{\phi}\left(g^{\mu \eta} g^{\rho \phi}-g^{\mu \rho} g^{\eta \phi}\right)\right] \psi=0 \quad .
\end{array}
$$


Thus, in the momentum space, we obtain, at the lowest nontrivial order, the following relationship:

$$
\begin{aligned}
& \left(p_{\mu} p^{\mu}+2 p_{\mu} c^{\mu \alpha} p_{\alpha}+2 p_{\mu} a^{\mu}-m^{2}\right)^{2}+4\left[p_{\mu} p^{\mu} d_{\rho}^{\beta} p_{\beta} d^{\rho \delta} p_{\delta}-\left(p_{\mu} d^{\mu \beta} p_{\beta}\right)^{2}\right] \\
+ & 8\left(p_{\mu} p^{\mu} d_{\eta}{ }^{\beta} p_{\beta} b^{\eta}-p_{\mu} d^{\mu \beta} p_{\beta} b_{\eta} p^{\eta}\right)+4\left[b_{\mu} b^{\mu} p_{\nu} p^{\nu}-\left(b_{\mu} p^{\mu}\right)^{2}\right]=0
\end{aligned}
$$

Hence, the dispersion relation arising from the Lorentz-violating extension of the SM is given by

$$
\begin{gathered}
p_{\mu} p^{\mu}-m^{2}=-2 p_{\mu} c^{\mu \alpha} p_{\alpha}-2 p_{\mu} a^{\mu} \pm 2\left[\left(p_{\mu} d^{\mu \beta} p_{\beta}\right)^{2}-p_{\mu} p^{\mu} d_{\eta}{ }^{\beta} p_{\beta} d^{\eta \delta} p_{\delta}\right. \\
\left.+\quad 2\left(p_{\mu} d^{\mu \beta} p_{\beta} b_{\rho} p^{\rho}-p_{\mu} p^{\mu} d_{\eta}^{\beta} p_{\beta} b^{\eta}\right)-b_{\mu} b^{\mu} p_{\nu} p^{\nu}+\left(b_{\mu} p^{\mu}\right)^{2}\right]^{1 / 2}
\end{gathered}
$$

where the \pm sign refers to the fact that the effects of $b_{\mu}$ and $d_{\mu \nu}$ depend on chirality.

Finally, considering, for simplicity, the scenario where coefficients $a_{\mu}, b_{\mu}, c_{\mu \nu}$, and $d_{\mu \nu}$ have only timelike components, it follows that the dispersion relation simplifies to

$$
p_{\mu} p^{\mu}-m^{2}=-2 c_{00} E^{2}-2 a E \pm 2\left(b+d_{00} E\right) p
$$

where we have dropped the component indices of coefficients $a$ and $b$. From now on we shall also drop coefficient $a$ as it may lead to changing flavour neutral currents when more than one flavour is involved.

In the next section, we shall use the dispersion relation Eq. (9) to see how the GZK cutoff for ultra-high energy cosmic rays can be relaxed. The following discussion is similar to the one described in [10], where it is assumed that the limiting velocities of particles in different reference frames are not the same.

\section{Ultra-High Energy Cosmic Rays in the Lorentz-violating Extention of the Standard Model}

The discovery of the cosmic background radiation has made raising the question of how the most energetic cosmic-ray particles would be affected by the interaction with the sea of microwave 
photons inevitable. In fact, the propagation of the ultra-high energy nucleons is limited by inelastic impacts with the ubiquitous photons of the background radiation disabling nucleons with energies above $5 \times 10^{19} \mathrm{eV}$ to reach Earth from further than $50-100 M p c$. This is the well known GZK cutoff [33]. However, events where the estimated energy of the cosmic primaries is beyond the GZK cutoff have been observed by different collaborations [34, 35, 36, 37]. It has been suggested [10] (see also [38) that slight violations of Lorentz invariance would cause energy-dependent effects which would suppress processes, otherwise dynamically inevitable, e.g. the resonant scattering reaction,

$$
p+\gamma_{2.73 K} \rightarrow \Delta_{1232}
$$

which is at the very core of the GZK cutoff. Were this process untenable, the GZK cutoff would not exist and consequently a cosmological origin for the high-energy cosmic radiation could be possible t. As discussed in [10], this can occur through a change in the dispersion relation for free particles. We shall see that this is indeed what happens when analyzing process Eq. (10) with dispersion relation Eq. (9). Considering a head-on impact of a proton of energy $E$ with a cosmic background radiation photon of energy $\omega$, the likelihood of the process Eq. (10) would be conditioned by Eq. (9) to be

$$
2 \omega+E \geq m_{\Delta}\left(1-c_{00}^{\Delta}\right)
$$

Hence, we get the following relationship, after squaring Eq. (11) and dropping the $\omega^{2}$ term:

$$
2 \omega+\frac{m_{p}^{2}}{2 E} \geq\left(c_{00}^{p}-c_{00}^{\Delta}\right) E+\frac{m_{\Delta}^{2}}{2 E}
$$

which clearly exhibits Lorentz-violating terms.

Let us now compare Eq. (12) with the results of Ref. [10] and show that this leads to a bound on $c_{00}^{i}$. To modify the usual dispersion relation for free particles, Coleman and Glashow suggest assigning a maximal attainable velocity to each particle. Therefore, for a given particle $i$ moving freely in the preferred frame, which could be thought of as the one in relation to which the cosmic

\footnotetext{
${ }^{4}$ Actually, it has been pointed out that the five highest-energy cosmic ray events seem to be closely correlated in space with cosmologically distant compact radio-loud quasars [39].
} 
background radiation is isotropic, the relevant dispersion relation would be

$$
E^{2}=p^{2} c_{i}^{2}+m_{i}^{2} c_{i}^{4}
$$

Hence the likelihood of the process Eq. (10) to occur under the conditions described above would depend on satisfying the kinematical condition $2 \omega+E \geq m_{e f f}$, where the effective mass $m_{e f f}$ is given by

$$
m_{e f f}^{2} \equiv m_{\Delta}^{2}-\left(c_{p}^{2}-c_{\Delta}^{2}\right) p^{2}
$$

the momentum being in respect to the preferred frame.

The likely condition takes then the following form

$$
2 \omega+\frac{m_{p}^{2}}{2 E} \geq\left(c_{p}-c_{\Delta}\right) E+\frac{m_{\Delta}^{2}}{2 E},
$$

where the term proportional to $c_{p}-c_{\Delta}$ is clearly Lorentz-violating. If the difference in the maximal velocities exceeds the critical value

$$
\delta(\omega)=\frac{2 \omega^{2}}{m_{\Delta}^{2}-m_{p}^{2}},
$$

then reaction Eq. (10) would be forbidden and consequently the GZK cutoff relaxed. For photons of the microwave background, $T=2.73 \mathrm{~K}$, and $\omega_{0} \equiv k T=2.35 \times 10^{-4} \mathrm{eV}$, this condition would be

$$
c_{p}-c_{\Delta}=\delta\left(\omega_{0}\right) \simeq 1.7 \times 10^{-25},
$$

which is quite a striking limit on the violation of the Lorentz symmetry, even though it is valid only for the process in question. Similar bounds for other particle pairs, although less stringent, were discussed in [10, 40].

Finally, thecomparison of Eq. (15) with Eq. (12) gives for $\Delta c_{00}$ :

$$
c_{00}^{p}-c_{00}^{\Delta} \simeq 1.7 \times 10^{-25}
$$

Thus, we see that the Lorentz-violating extension of the SM can explain the violation of the GZK cutoff and account for the phenomenology of ultra-high energy cosmic rays via the bound on $\Delta c_{00}$ given by Eq. (18). Of course, the situation would be more complex if the Lorentzviolating parameters were allowed to have spacelike components which would lead to direction and helicity-dependent effects. 


\section{An astrophysical test of Lorentz Invariance}

Let us turn to the discussion of a possible astrophysical test of Lorentz invariance. From eq. (18) we see that $\Delta c_{00} \simeq \epsilon$, where $\epsilon$ is a small constant specific of the process involved (cf. eq. (18)) and, for instance, $|\epsilon| \lesssim f e w \times 10^{-22}$ from the search of neutrino oscillations 41, 42]. In what concerns signals simultaneously emitted by faraway sources, the resulting effect in the propagation velocity of particles with energy, $E$, and momentum, $p$, is given in the limit where $m<<p, E$ or for massless particles by $c_{i}=c\left[1-\left(c_{00} \pm d_{00}\right)_{i}\right]$. Hence, for sources at a distance $D$, the relative delay in the arrival time will be given by

$$
\Delta t \simeq \frac{D}{c}\left[\left(c_{00} \pm d_{00}\right)_{i}-\left(c_{00} \pm d_{00}\right)_{j}\right] \equiv \epsilon_{i j}^{ \pm} \frac{D}{c} \quad,
$$

where we have defined a new constant, $\epsilon_{i j}^{ \pm}$, involving a pair of particles. This time delay may, despite being given by the difference between two quite small numbers, be measurable for sufficiently far away sources. Moreover, our result indicates that the estimated time delay is energy independent, in opposition to what one could expect from general arguments [5, 7, 8]. We have also found that the time delay has an interesting dependence on the chirality of the particles involved. In the next section, we shall discuss how to estimate the scales involved in the observational value of $c_{00}^{i}\left(\right.$ and $d_{00}^{i}$ if $\left.d_{00}^{i} \sim c_{00}^{i}\right)$.

Therefore if, for instance, the signals from faraway sources were, as suggested in the introduction, from an AGN TeV gamma-ray flare and the genetically related neutrino emission, then we should expect for the time delay, if as justified above that the photon propagation is unaltered,

$$
\Delta t \simeq\left(c_{00} \pm d_{00}\right)_{\nu} \frac{D}{c}
$$

assuming that the neutrinos are massless, an issue that will hopefully be settled experimentally in the near future. It is worth stressing that even before that, the effect of neutrino masses and other intrinsic effects related to the nature of the neutrino emission processes can, at least in principle, be extracted from the data of several correlated detections of $T e V$ gamma-ray flares and neutrinos if a systematic delay of neutrinos is observed. However, the main point here is that a nonvanishing time delay can be regarded, up to neutrino mass effects and neutrino emission processes, as direct evidence for a violation of Lorentz symmetry and, as already pointed out 
in the introduction, neutrino telescopes will soon be available for the investigation of correlated detections. Furthermore, the available knowledge of AGN phenomena and our confidence in the astrophysical methods available to determine their distance from us make it reasonable to believe that the time delay strategy may realistically provide relevant limits on the violation of Lorentz symmetry. Of course, the same arguments may very well apply to GRB; however, the lack of a deeper understanding of these transient phenomena introduces further unnecessary uncertainty, even though many properties of their sources can be understood from the observation of their afterglows. It is also important to point out that limits involving photons and neutrinos are currently unknown and that a difference between neutrinos and antineutrinos is expected if $d_{00}^{i}$ is nonvanishing. We could also add that, based on the analysis of Ref. [27] involving the full set of Lorentz-violating parameters, we expect our results to remain unaltered, at least at high energies, if the parameter $H_{\mu \nu}$ were to be held in our calculations.

\section{Discussion and Conclusions}

In summary, we have shown that parameters of the Lorentz-violating extension of the SM proposed in Ref. [9] can be related to the phenomenology of ultra-high energy cosmic rays with the conclusion that, as in [10], it may lead to the suppression of processes responsible for the GZK cutoff. This is a crucial argument for an extragalactic origin of high-energy cosmic rays. Fur-

thermore, we have found that the relevant Lorentz-violating parameter is, at high energies, $c_{00}^{i}$ so that $\Delta c_{00} \simeq \epsilon$ with $|\epsilon| \lesssim f e w \times 10^{-22}$ from neutrino physics and $|\epsilon| \lesssim 10^{-25}$ from the ultra high-energy cosmic-ray physics. Actually, it is possible to estimate the typical scales involved in the problem assuming that the source of Lorentz symmetry violation is due to nontrivial solutions in string field theory. Indeed, these solutions imply that Lorentz tensors acquire vacuum expection values as Lorentz symmetry is spontaneously broken due to string-induced interactions [14, 17]. A sensible parametrization for these expectation values and hence for $c_{00}^{i}$ would be the following for a fixed energy scale, E, 17, 22]:

$$
c_{00}^{i} \simeq \frac{\langle T\rangle}{M_{S}}=\lambda_{i}\left(\frac{m_{L}}{M_{S}}\right)^{l}\left(\frac{E}{M_{S}}\right)^{k},
$$


where $T$ denotes a generic Lorentz tensor, $\lambda_{i}$ is presumably an order one flavour-dependent constant $m_{L}$ is a light mass scale, $M_{S}$ is a string scale presumably close to Planck's mass or a few orders of magnitude below it, and $k, l$ are integers indicating the order of the string corrections to low-energy physics. Thus, in the lowest non-trivial order, $k=0, l=1(k=l=0$ is already excluded experimentally), $c_{00}^{i}=\lambda_{i}\left(\frac{m_{L}}{M_{S}}\right)$ and for different $\lambda_{i}$ constants $\epsilon \simeq\left(\frac{m_{L}}{M_{S}}\right)$. This result corresponds, in its essential lines, to the one we have obtained from working out the implications of the Lorentz-violating extension of the SM in the context of ultra-high energy cosmic-ray phenomenology. Furthermore if, for instance, $\epsilon \lesssim 10^{-23}$, then it follows that $m_{L} \sim 10^{2} \mathrm{KeV}$ for $M_{S} \simeq M_{P}$ or $m_{L} \sim 10^{2} \mathrm{eV}$ if $M_{S} \simeq \mathrm{few} \times 10^{16} \mathrm{GeV}$ [43]. Estimates for $m_{L}$ would clearly change by many orders of magnitude if $\lambda_{i}$ were of the order of the Yukawa coupling. In either case, our main conclusion is that choice $k=0, l=1$ implies that the time delay in the arrival of signals from faraway sources is, as discussed above, energy independent. We have found, however, an interesting dependence on the chirality of particles involved.

Naturally, another scenario would emerge from a different choice of integers $k, l$. For instance, the choice $k=2$ and $l=0$, the relevant choice in the CPT symmetry violating baryogenesis scenario [22], where the energy should, in this case, be related to the early Universe temperature. This would imply that the time delay in the arrival of signals from faraway sources would be proportional to the square of the energy. This choice would also lead to the conclusion that Lorentz-violating effects, whether due to string physics or quantum gravity, are quadratic in the energy. Interestingly, similar conclusions concerning the order of quantum gravity low-energy effects are reached from the study of corrections to the Schrödinger equation arising from quantum gravity in the mini-superspace approximation [44].

Another setting allowing for the spontaneous breaking of the Lorentz symmetry is the so-called braneworld [31]. In this scenario, SM particles lie on a three-brane, $\phi(x)$, embedded in spacetime, with possibly large compact extra dimensions, whereas gravity propagates in the bulk. Thus, a tilted brane induces rotational and Lorentz noninvariant terms in the four-dimensional effective theory as brane-Goldstones couple to all particles on the brane via an induced metric on the brane.

\footnotetext{
${ }^{5}$ Another scenario would be for $\lambda_{i}$ to be of the order of the respective Yukawa coupling [17].
} 
This will lead to operators of the form

$$
\partial_{\mu} \phi \partial_{\nu} \phi \bar{\psi} \gamma^{\mu} \partial^{\nu} \psi+\partial^{\mu} \phi \partial_{\nu} \phi F_{\mu \alpha} F^{\nu \alpha} \ldots
$$

which closely resemble the Lorentz-violating terms in the SM extention. As before, phenomenology sets tight constraints on this scenario, which is however, currently unable to establish whether the breaking of Lorentz invariance, if observed at all, has its origin in the nonperturbative nature of branes or if it arises from the perturbative string field theory scenario described above. The former

alternative could possibly be associated with a $M_{S}$ scale that is a few orders of magnitude below the Planck scale, while the latter with a $M_{S}$ that should be associated with the Planck scale itself.

Finally, we could say that based on our results, we have outlined a strategy to establish to what extent Lorentz invariance is violated from the observation of the time delay in the detection of $\mathrm{TeV}$ gamma-ray flares and neutrinos from AGN. Our analysis reveals that the time delay has a dependence on the chirality of the particles involved, but is energy independent, contrary to what one could expect from general arguments. In either case, if ever observed, a time delay in the arrival of signals from faraway sources would be, up to neutrino mass effects and other features related to the nature of the neutrino emission, strong evidence of quite new physics beyond the SM.

\section{Acknowledgments}

One of us (O.B.) would like to thank the hospitality of Department of Physics of the New York University where part of this work was carried out and Fundação para a Ciência e a Tecnologia (Portugal) for the financial support under grant No. BSAB/95/99. It is a pleasure to thank Alan Kostelecký for important comments and suggestions. We also thank A. Halprin for comments. 


\section{References}

[1] K. Mannheim, Astropart. Phys. 3 (1995) 295;

F. Halzen and E. Zas, Ap. J. 488 (1997) 669.

[2] E. Waxman and J. Bahcall, "High Energy Neutrinos from Astrophysical Sources: An Upper Bound", hep-ph/9807282.

[3] E. Waxman and J. Bahcall, Phys. Rev. Lett. 78 (1997) 2292.

[4] T.D. Gaisser, F. Halzen and T. Stanev, Phys. Rep. 258 (1995) 173.

[5] G. Amelino-Camelia, J. Ellis, N.E. Mavromatos, D.V. Nanopoulos and S. Sarkar, Nature 393 (1998) 763 .

[6] S.D. Biller et al., "Limits to Quantum Gravity Effects from Observations of $T e V$ Flares in Active Galaxies", gr-qc/9810044.

[7] G. Amelino-Camelia, J. Ellis, N.E. Mavromatos, D.V. Nanopoulos and S. Sarkar, "Sensitivity of Astrophysical Observations to Gravity-Induced Wave Dispersion in Vacuo", astroph/9810483.

[8] J. Ellis, K. Farakos, N.E. Mavromatos, V.A. Mitsou and D.V. Nanopoulos, "Astrophysical Probes of the Constancy of the Velocity of Light", astro-ph/9907340.

[9] D. Colladay and V.A. Kostelecký, Phys. Rev. D55 (1997) 6760; D58 (1998) 116002.

[10] S. Coleman and S.L. Glashow, Phys. Lett. B405 (1997) 249; Phys. Rev. D59 (1999) 116008.

[11] P.R. Phillips, Phys. Rev. 146 (1966) 966.

[12] H.B. Nielsen and M. Ninomiya, Nucl. Phys. B141 (1978) 153.

[13] S. Weinberg, Phys. Lett. B138 (1984) 47.

[14] V.A. Kostelecký and S. Samuel, Phys. Rev. D39 (1989) 683; Phys. Rev. Lett. 63 (1989) 224.

[15] O. Bertolami, Class. Quantum Gravity 14 (1997) 2748. 
[16] O. Bertolami and D.F. Mota, Phys. Lett. B455 (1999) 96.

[17] V.A. Kostelecký and R. Potting, Phys. Rev. D51 (1995) 3923; Phys. Lett. B381 (1996) 389.

[18] D. Colladay and V.A. Kostelecký, Phys. Lett. B344 (1995) 259; Phys. Rev. D52 (1995) 6224; V.A. Kostelecký and R. Van Kooten, Phys. Rev. D54 (1996) 5585.

[19] R. Adler et al. (CPLEAR Collaboration), J. Ellis, J.L. Lopez, N.E. Mavromatos and D.V. Nanopoulos, Phys. Lett. B364 (1995) 239.

[20] R. Bluhm, V.A. Kostelecký and N. Russell, Phys. Rev. Lett. 79 (1997) 1432; Phys. Rev. D57 (1998) 3932 .

[21] R. Bluhm, V.A. Kostelecký and N. Russell, Phys. Rev. Lett. 82 (1999) 2254.

[22] O. Bertolami, D. Colladay, V.A. Kostelecký and R. Potting, Phys. Lett. B395 (1997) 178.

[23] A. Brillet and J.L. Hall, Phys. Rev. Lett. 42 (1979) 549.

[24] V.W. Hughes, H.G. Robinson and V. Beltran-Lopez, Phys. Rev. Lett. 4 (1960) 342.

[25] R.W.P. Drever, Philos. Mag. 6 (1961) 683.

[26] S.K. Lamoreaux, J.P. Jacobs, B.R. Heckel, F.J. Raab and E.N. Fortson, Phys. Rev. Lett. 57 (1986) 3125 .

[27] V.A. Kostelecký and C.D. Lane, Phys. Rev. D60 (1999) 116010.

[28] C.M. Will, "Theory and Experiment in Gravitational Physics" (Cambridge University Press, 1993).

[29] J.F. Bell, Ap. J. 462 (1996) 287.

[30] J.F. Bell and T. Damour, Class. Quantum Gravity 13 (1996) 3121.

[31] G. Dvali and M. Shifman, "Tilting the Brane, or Some Cosmological Consequences of the Brane World", hep-th/9904021. 
[32] S.M. Carroll, G.B. Field and R. Jackiw, Phys. Rev. D41 (1990) 1231;

S.M. Carroll and G.B. Field, Phys. Rev. Lett. 79 (1997) 2394.

[33] K. Greisen, Phys. Rev. Lett. 16 (1966) 748;

G.T. Zatsepin and V.A. Kuzmin, JETP Lett. 41 (1966) 78.

[34] N. Hayashida et al., (AGASA Collab.), Phys. Rev. Lett. 73 (1994) 3491;

M. Takeda et al., (AGASA Collab.), Phys. Rev. Lett. 81 (1998) 1163.

[35] D.J. Bird et al., (Fly's Eye Collab.), Phys. Rev. Lett. 71 (1993) 3401; Ap. J. 424 (1994) 491; 441 (1995) 144.

[36] M.A. Lawrence, R.J.O. Reid and A.A. Watson (Haverah Park Collab.), J. Phys. G17 (1991) 733.

[37] N.N. Efimov et al., (Yakutsk Collab.), ICRR Symposium on Astrophysical Aspects of the Most Energetic Cosmic Rays, eds. N. Nagano and F. Takahara (World Scientific, 1991).

[38] L. Gonzales-Mestres, "Deformed Lorentz Symmetry and Ultra-High Energy Cosmic Rays ", hep-ph/9905430.

[39] G.R. Farrar and P.L. Biermann, Phys. Rev. Lett. 81 (1998) 3579.

[40] A. Halprin and H.B. Kim, "Mapping Lorentz Invariance Violations into Equivalence Principle Violations", hep-ph/9905301.

[41] E.B. Brucker et al., Phys. Rev. D34 (1986) 2183.

[42] S.L. Glashow, A. Halprin, P.I. Krastev, C.N. Leung and J. Pantaleone, Phys. Rev. D56 (1997) 2433.

[43] E. Witten, Nucl. Phys. B471 (1996) 135.

[44] O. Bertolami, Phys. Lett. A154 (1991) 225. 